\title{
PREDICTION OF THE MECHANICAL BEHAVIOR OF A MINICOMPOSITE BASED ON GREY VERHULST MODELS
}

\author{
"YIQIANG WANG*, LAIFEI CHENG**, LITONG ZHANG** \\ *College of Aeronautical Engineering, Civil Aviation University of China, \\ Tianjin 300300, China \\ **National Key Laboratory of Thermostructure Composite Materials, Northwestern Polytechnical University, \\ Xi'an Shaanxi, China \\ "E-mail:wyq80@126.com
}

Submitted April 13, 2017; accepted June 2, 2017

\begin{abstract}
Keywords: C/SiC, Tensile behavior, Acoustic emission, Grey systems
The tensile behavior of a $\mathrm{C} / \mathrm{SiC}$ minicomposite fabricated by chemical vapor infiltration was examined and the associated damage evolution was monitored by using acoustic emission (AE) technique. Both the tensile behavior and the associated $A E$ evolution of the minicomposites can be well characterized by three regimes including matrix cracking emergence, multiplication followed by matrix cracking saturation. The grey Verhulst model and inverse Verhulst model were established for the evolution of the cumulative AE energy and the stress-strain behavior, respectively. Excellent agreement between predictions and experimental results was obtained for both the grey models, which indicates the nature of intraspecific competition for these matrix cracks in the minicomposites studied. Such robust relationships can be further applied to model the constitutive behaviour of other ceramic composite systems.
\end{abstract}

\section{INTRODUCTION}

Ceramic matrix composites (CMCs) have been proposed for decades as thermostructrual materials suitable for aerospace and aeronautical engine hotsection components like nozzle extensions, air mixers, etc. $[1,2]$. However, most current CMCs consist of multiple tows structured in different directions and complex arrangements such as woven, braided, needled and stitched to meet the mechanical requirements along certain directions [3]. The densification of these multi-dimensional fiber performs is essentially time consuming, regardless of the fabrication techniques, e.g. chemical vapour infiltration (CVI) or polymer impregnation and pyrolysis (PIP). Thus the mechanical modeling and processing optimization for these CMCs seems to be very inconvenient.

Fortunately, a cost-effective approach has been developed most recently by using minicomposites [4]. A large number of specimens can be fabricated at relatively low cost with short time, which makes it attractive for obtaining a robust set of experimental data. Since the minicomposite represents a subelement (infiltrated longitudinal bundles) of these real macrocomposites in loading direction, the tensile behaviour of minicomposites is believed to mimic that of their multidirectional counterparts, because the interfacial and elastic properties of the constituents are the same.
Up to now this approach has been used to study the mechanical/fatigue properties of CMCs with different commercial fibers including Nicalon and Hi-Nicalon at room/elevated temperatures [5-9]. However, most previous studies were mainly focused on SiC-fiber-reinforced composite systems, whereas the mechanical behavior and the corresponding modeling of $\mathrm{C} / \mathrm{SiC}$ minicomposites has been little reported. As a new method, the grey system theory is suitable for studying uncertain problems with less data and poor information. The application scope of grey system theory has extended to industry, social affairs, economy, energy, financial and other fields [10-12]. However, there are no efforts dealing with the mechanical behavior of a minicomposite based on the grey system. Therefore, a $\mathrm{C} / \mathrm{SiC}$ minicomposite was fabricated in the present work, and then tensile tested at room temperature, while the mechanical behavior was studied and predicted based on the grey system with the aid of the acoustic emission (AE) technique.

\section{EXPERIMENTAL}

\section{Composite fabrication}

The carbon fiber utilized was T300 (Nippon Toray Co., Japan), and each bundle contained 3000 fibers (with a filament diameter of $7 \mu \mathrm{m}$ ). The continuous 
as-received multifilament bundle was wrapped on a graphite frame with a width of $80 \mathrm{~mm}$ to ensure that the gauge length of minicomposites is at least $50 \mathrm{~mm}$. The $\mathrm{C} /$ $\mathrm{SiC}$ minicomposite was fabricated by isothermal CVI to deposit the pyrocarbon $(\mathrm{PyC})$ interphase and subsequent $\mathrm{SiC}$ matrix. Methyltrichlorosilane (MTS, $\mathrm{CH}_{3} \mathrm{SiCl}_{3}$ ) was used for the deposition of the SiC matrix. MTS vapor was carried by bubbling hydrogen. The processing conditions for minicomposites are the same as for the real macrocomposites, except that the infiltration time of $\mathrm{SiC}$ matrix is about $1 / 4$ of that of macrocomposites in order to avoid over-thick $\mathrm{SiC}$ sheath around the minicomposites. The volume fractions of fiber of each specimen were determined from the weights measured on minicomposites and on reference fiber bundles, leading to an average volume fraction of fibers $\mathrm{V}_{\mathrm{f}} \approx 0.3$. The ends of specimens were mounted with epoxy to aluminum tabs. An alligator clip on every side of the tabs was used to facilitate handling of specimens until its removal before tensile tests. Much care was taken to avoid the premature or unexpected failure of the specimens.

\section{Tension tests and microstructural observation}

Tensile testing was performed on a fiber testing machine (Model 3345, Instron, USA) with a load cell of $1 \mathrm{kN}$. Tensile loads were applied on the minicomposites by the two pins fixed with the crosshead of machine through the holes of aluminum tabs. The minicomposite stress was determined by dividing the applied load by the calculated minicomposite cross-sectional area. The crosshead speed is $0.5 \mathrm{~mm} / \mathrm{min}$ and 10 specimens were tested. Tensile strains were measured by a non-contact optical strain gauge with the gauge length of $50 \mathrm{~mm}$.

The activities of the acoustic emission were monitored with an AE sensor (WD, PhysicalAcoustic Corpo- ration, USA) which was attached to the aluminum tabs. Vacuum grease was used as a couplant. A PCI-2 (PhysicalAcoustic Corporation, USA) AE system was used to analyze and store the signals that were captured by the sensor. Both the preamplifier gain and the threshold were set at $40 \mathrm{~dB}$. All signals were frequency-filtered so that only frequencies between 20 and $2000 \mathrm{kHz}$ were used for further processing. A counterweight was also used to eliminate the possible bending moments near the ends of specimens due to the attachment of $\mathrm{AE}$ sensor to the tabs. The failed minicomposites were mounted in epoxy, and then polished longitudinally to a $1 \mu \mathrm{m}$ finish to determine the matrix-crack spacing.

\section{RESULTS AND DISCUSSION}

\section{Microstructure characterization}

A typical cross-section of $\mathrm{C} / \mathrm{SiC}$ minicomposites is shown in Figure 1. It can be seen that the cross-section is close to elliptical, with the relatively thick CVI-SiC sheath surrounding the bundle. It can also be seen from the longitudinal surface as shown in Figure 2 that the outer $\mathrm{SiC}$ sheath was actually formed by relatively uniform $\mathrm{SiC}$ particles with the diameter of about 100 $\mu \mathrm{m}$ which were finally deposited by the current CVI process. Based on the image analysis using Photoshop software, the minicomposites studied have a cross-sectional area of $0.35-0.42 \mathrm{~mm}^{2}$. This is consistent with the results determined from the weights measured on minicomposites and on reference fiber bundles.

After tensile tests, an example of polished longitudinal gauge sections of $\mathrm{C} / \mathrm{SiC}$ minicomposites was shown in Figure 3. It is evident that the multiple matrix cracks crossing the entire minicomposites are densely spaced and of relatively uniform intervals, which is indicative of the matrix cracking saturation achieved for the minicomposites studied.

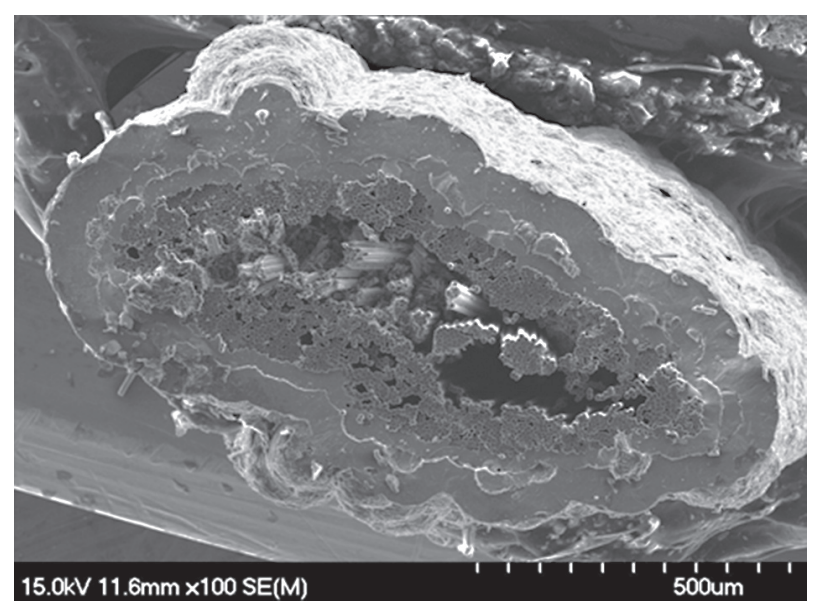

a) Overview of the specimen contour

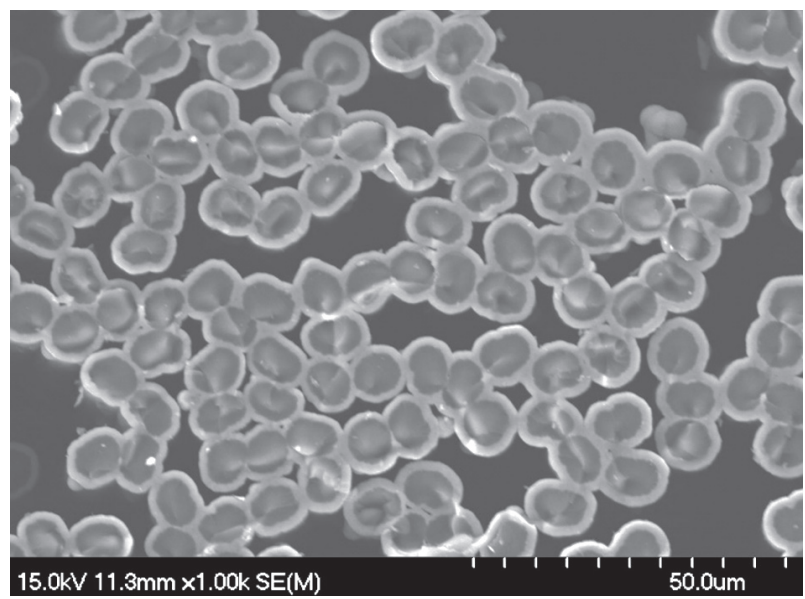

b) Magnification view of the inside

Figure 1. Typical micrograph of the cross-section of minicomposites. 


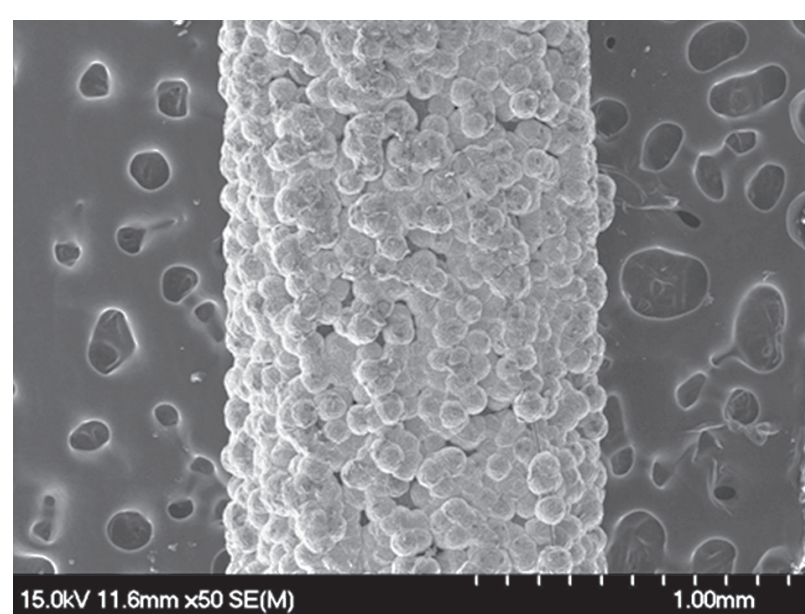

Figure 2. Longitudinal observations of as-received minicomposites showing uniform $\mathrm{SiC}$ particles deposited.

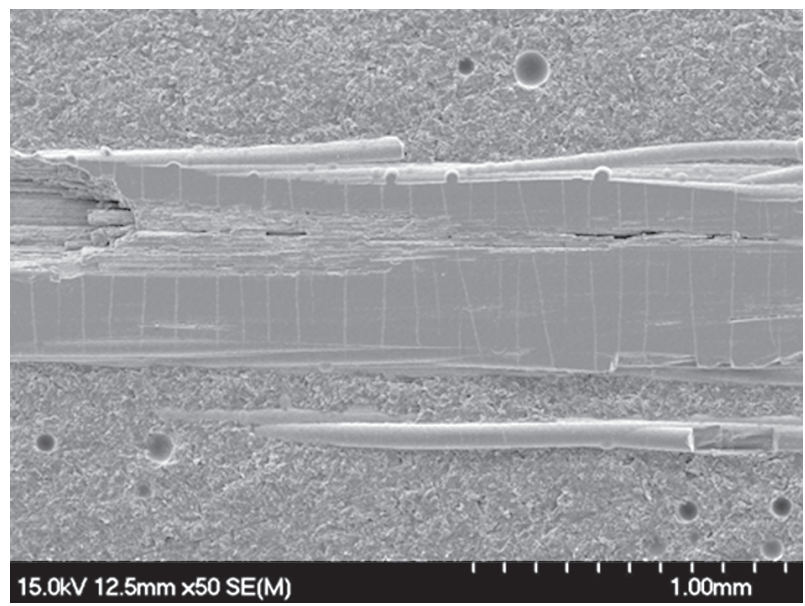

Figure 3. Longitudinal observations of tested minicomposites showing densely spaced matrix cracks.

Tension test results

The test results on this batch of minicomposites show few scatters and the typical tensile stress-strain curve, along with the corresponding curve of cumulative AE energy and AE energy per event, is shown in Figure 4. The tensile behavior of the minicomposites is largely nonlinear from the onset of loading. Three different domains corresponding to three $\mathrm{AE}$ different steps may be noticed from both the stress-strain curve and the curve of cumulative AE energy versus strain. The first domain is characterized by a slow increase in the accumulation of AE energy with low-energy events occurred. This corresponds to the processing-induced defects or microcracks due to thermal residual stresses in $\mathrm{C} / \mathrm{SiC}$ minicomposites cooled from processing temperature $\left(>1000^{\circ} \mathrm{C}\right)$ to room temperature, which extend but not across, at least not very much, the minicomposites.

A second domain is characterized by a rapid increase in the rate of accumulation of AE energy due to dense high-energy events occurred that correspond to the large matrix cracks newly formed and/or propagating throughthe-diameter of the minicomposites, i.e. multiplication of the matrix microcracking and associated interface debonding until saturation.

The last part of the stress-strain curve displays a slope recovery and the rate of cumulative AE energy actually starts to diminish to nearly plateau with increasing applied load until fracture. This corresponds to the matrix cracking saturation and elongation of the remained intact fibers, which are carrying the entire load. Rupture then occurs at an average stress of $350 \mathrm{MPa}$ for a corresponding average strain of $0.66 \%$.

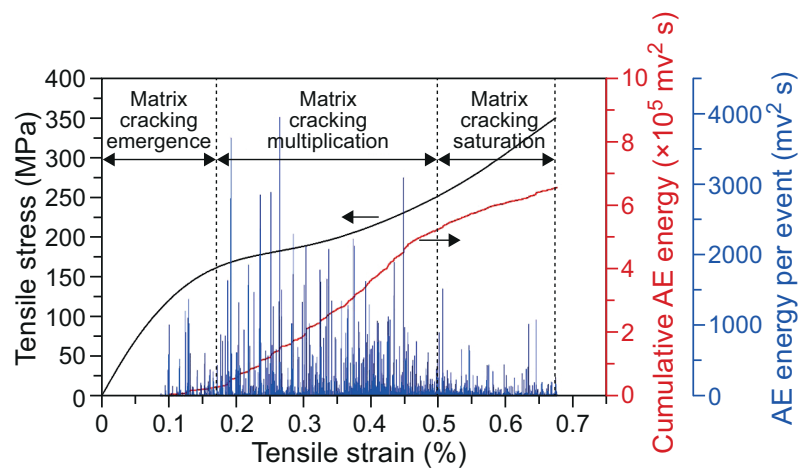

Figure 4. Typical tensile stress-strain curve with the associated AE activities.

The above-mentioned domains exhibited in both the stress-strain curve and the accumulation of $\mathrm{AE}$ energy curve including matrix cracking emergence, multiplication and saturation, are very similar to the development of the self-limiting growth of a biological population, which is often described by the Verhulst equation, namely the law of population growth as follows

$$
\frac{\mathrm{d} N}{d t}=r N-\alpha N^{2}
$$

where $N$ represents number of individuals at time $t, r$ the intrinsic growth rate, and $\alpha$ is the density-dependent crowding effect (also known as intraspecific competition). In this equation, the population equilibrium (the so-called carrying capacity) $K$ can be derived as

$$
K=\frac{r}{\alpha}
$$

In the minicomposites studied, the early, unimpeded matrix crack growth rate can be modeled by the first term $r N$. The value of the rate $r$ represents the proportional increase of the crack number $N$. Later, as the damages evolve, the effect of the second term becomes almost as large as the first, as some members of the matrix cracks interfere with each other by competing for the critical matrix space. This antagonistic effect is called the bottleneck, and can be modeled by the value of the parameter of the carrying capacity. The competition 
diminishes the combined growth rate, until the number of the matrix cracks ceases to grow, i.e. the domain of the matrix cracking saturation corresponding to the so-called maturity of the population.

Since the amount of cumulative AE energy is nearly directly related to the number of matrix cracks formed, it can also be described by the Verhulst equation as follows

$$
\frac{\mathrm{d} E^{(1)}}{\mathrm{d} \varepsilon}=a E^{(1)}-b E^{(1)^{2}}
$$

where $E^{(l)}$ is the amount of cumulative AE energy, $\varepsilon$ is the tensile strain. Thus its grey difference equation in the discrete form can be described as

$$
E_{(k)}^{(0)}=a z_{(k)}^{(1)}-b z_{(k)}^{(1)^{2}}
$$

where $E_{(k)}^{(0)}=\left[E_{(k)}^{(1)}-E_{(k-1)}^{(1)}\right] /\left[\varepsilon_{(k)}-\varepsilon_{(k-1)}\right], k$ is the sequential number of measured data and $k=2,3, \ldots, n$. And the sequence $Z(k)$ can be obtained by using the mean consecutive neighbors operator for $E^{(l)}$ as follows

$$
Z(k)=1 / 2\left[E_{(k)}^{(1)}+E_{(k-1)}^{(1)}\right], k=2,3, n .
$$

The parameters of $a$ and $b$ can be estimated as follows by minimizing the squared-errors, i.e., least squares method:

$$
\left[\begin{array}{l}
\hat{a} \\
\hat{b}
\end{array}\right]=\left(B^{T} B\right)^{-1} B^{T} Y
$$

where

$$
\begin{aligned}
& B=\left[\begin{array}{cc}
z(2) & -z(2)^{2} \\
\cdots & \cdots \\
z(n) & -z(n)^{2}
\end{array}\right] \\
& Y=\left[\begin{array}{c}
E_{(2)}^{(0)} \\
\cdots \\
E_{(n)}^{(0)}
\end{array}\right]
\end{aligned}
$$

Based on the estimated parameters, the AE energy response function can be obtained by solving Equation (3), as shown below:

$$
E_{(k)}^{(1)}=\frac{a / b}{1+\left(\frac{a 1}{b_{E_{(1)}^{(1)}}}-1\right) \mathrm{e}^{-a[\varepsilon(k)-\varepsilon(1)]}}
$$

where the initial condition of the model is set as $\varepsilon=\varepsilon(1)$, $E=E_{(1)}^{(1)}$, namely using the first measured data point.

The measured cumulative AE energy versus strain at fixed intervals are listed in Table 1. The strain can be normalized by the true tensile strain divided by $0.05 \%$ for mathematical convenience, leading to $\varepsilon(\mathrm{k})$ equal to the sequence number $k$.

Therefore the response of the cumulative AE energy versus strain can be predicted as follows based on the grey verhulst model above and experimental data in Table 1,

$$
E_{(k)}^{(1)}=\frac{6.81}{1+22.49 e^{-0.4838(k-1)}}
$$

where $a=0.4838, b=0.071$.
Table 1. Measured cumulative AE energy at regular strain intervals.

\begin{tabular}{ccc}
\hline $\begin{array}{c}\text { Sequence } \\
\text { number }\end{array}$ & $\begin{array}{c}\text { Tensile strain } \\
(\%)\end{array}$ & $\begin{array}{c}\text { AE energy } \\
\left(\times 10^{5} \mathrm{mV}^{2} \cdot \mathrm{s}\right)\end{array}$ \\
\hline 1 & 0.1 & 0.29 \\
2 & 0.15 & 0.81 \\
3 & 0.2 & 1.48 \\
4 & 0.25 & 2.12 \\
5 & 0.3 & 2.78 \\
6 & 0.35 & 3.51 \\
7 & 0.4 & 4.18 \\
8 & 0.45 & 5.14 \\
9 & 0.5 & 5.59 \\
10 & 0.55 & 5.93 \\
11 & 0.6 & 6.23 \\
12 & 0.65 & 6.53 \\
\hline
\end{tabular}

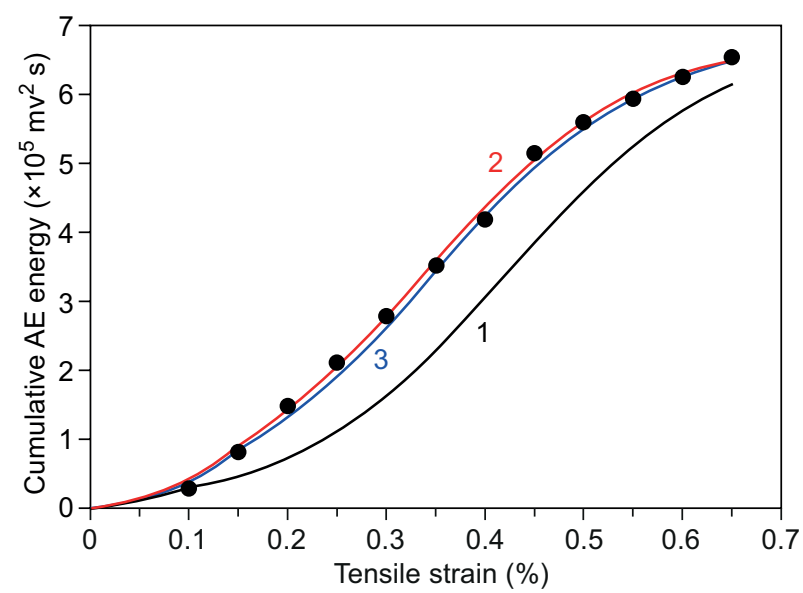

Figure 5. Evolutions of the cumulative AE energy with tensile strains. Experimental data are represented by black circles, while curve 1 represents the prediction by grey Verhulst model without optimized initial condition, curve 2 with optimized initial condition and curve 3 with the initial condition set through the fifth data point.

The predicted cumulative AE energy curve is shown in Figure 5 along with the experimental data. It can be seen that an evident deviation exits between the prediction and experimental data except the first point. This is due to an improper setting of initial condition to Equation 3, since there is no sufficient evidence that using the first raw data would lead to higher prediction accuracy. Consequently the initial condition should be optimized to achieve a better agreement. For convenience the Equation 9 can be rewritten as

$$
\widehat{E_{(k)}^{(1)}}=\frac{1}{\frac{b}{a}+c e^{-a[\varepsilon(k)-\varepsilon(1)]}}
$$

where $\widehat{E_{(k)}^{(1)}}$ is the predicted cumulative AE energy, $c$ is the parameter reflecting the initial condition, which can be optimized by minimize the sum of squared errors of the reciprocals as follows 


$$
\begin{aligned}
\min Q & =\min \sum_{k=2}^{n}\left(\widehat{\frac{1}{E_{(k)}^{(1)}}}-\frac{1}{E_{(k)}^{(1)}}\right)^{2} \\
& =\min \sum_{k=2}^{n}\left(\frac{b}{a}+c e^{-a[\varepsilon(k)-\varepsilon(1)]}-\frac{1}{E_{(k)}^{(1)}}\right)^{2}
\end{aligned}
$$

The optimized parameter $c$ can be obtained through

$$
\frac{\mathrm{d} Q}{\mathrm{~d} c}=0
$$

And the result is

$$
c=\frac{\sum_{k=2}^{n}\left(\frac{1}{E_{(k)}^{(1)}}-\frac{b}{a}\right) e^{-a[\varepsilon(k)-\varepsilon(1)]}}{\sum_{k=2}^{n} e^{-2 a[\varepsilon(k)-\varepsilon(1)]}}
$$

Based on the measured data in Table 1, the parameter $c$ is finally optimized as 1.6289 , and the corresponding curve is also shown in Figure 5. It can be seen that the predicted curve exhibits an excellent agreement with the actual data. Furthermore, the prediction accuracy can also be improved by another simple method, where the initial condition is set as each measured data point, i.e. not only the first measured data point. Thus the initial condition can be finally determined after comparison of the fitting degrees. As shown in Figure 5, an excellent agreement was also achieved by the predicted curve with the initial condition set as $\varepsilon=\varepsilon(5), E=E_{(5)}{ }^{(1)}$

Since the energy of AE has shown to be a good measure of matrix cracking for CMCs, the stress-strain constitutive behavior seems to be very clearly a reflection of the AE energy curve. It was also confirmed by the three corresponding domains shown in both the curves. The domain of matrix cracking emergence with a slow

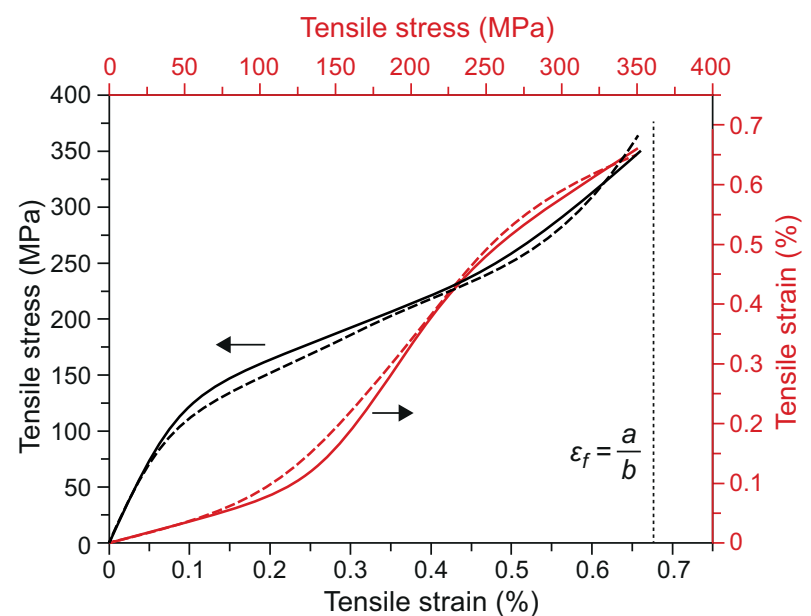

Figure 6. Prediction and measured constitutive behavior of the minicomposites. Experimental data are represented by solid lines, while curves with dash lines indicate the predictions by grey Verhulst model and inverse model. Also shown is the predicted carrying capacity of strain. increase of AE energy corresponds to the first domain of the stress-strain curve with a slightly nonlinear behavior. The domain of matrix cracking multiplication with a rapid increase of $\mathrm{AE}$ energy corresponds to the middle domain of the stress-strain curve with a largely nonlinear behavior. And the domain of matrix cracking saturation with a near plateau of AE energy corresponds to the final domain of the stress-strain curve with an obvious slope recovery. Consequently, the constitutive law of the minicomposites in this paper is also subject to the Verhulst equation, which is confirmed by strains plotted as a function of tensile stress as shown in Figure 6. It is evident that the strain-stress curve exhibits the very similar evolution to that of the AE energy curve. Therefore the grey verhulst method can be also used to model the constitutive law as follows

$$
\frac{\mathrm{d} \varepsilon^{(1)}}{\mathrm{d} \sigma}=a \varepsilon^{(1)}-b \varepsilon^{(1)^{2}}
$$

And the final constitutive law can be described as

$$
\varepsilon_{(k)}^{(1)}=\frac{a / b}{1+\left(\frac{a 1}{b_{\varepsilon_{(1)}^{(1)}}}-1\right) \mathrm{e}^{-a[\sigma(k)-\sigma(1)]}}
$$

where $k$ is also the sequential number and $\sigma_{(k)}$ was normalized by the true tensile stress divided by $50 \mathrm{MPa}$, leading to $\sigma_{(k)}$ equal to $k$.

Table 2. Measured tensile strain-stress data in equidistant stress sequence.

\begin{tabular}{ccc}
\hline $\begin{array}{c}\text { Sequence } \\
\text { number }\end{array}$ & $\begin{array}{c}\text { Tensile strain } \\
(\%)\end{array}$ & $\begin{array}{c}\text { Tensile stress } \\
(\mathrm{MPa})\end{array}$ \\
\hline 1 & 0.03 & 50 \\
2 & 0.07 & 100 \\
3 & 0.13 & 150 \\
4 & 0.34 & 200 \\
5 & 0.49 & 250 \\
6 & 0.58 & 300 \\
7 & 0.66 & 350 \\
\hline
\end{tabular}

Based on the measured sequential strain-stress data in Table 2, the parameters of $a$ and $b$ were estimated as

$$
\left[\begin{array}{l}
\hat{a} \\
\hat{b}
\end{array}\right]=\left[\begin{array}{l}
1.0382 \\
1.5262
\end{array}\right]
$$

Finally, the stress-strain curve can be obtained by the inverse function of Verhulst equation and described as

$$
\sigma=50\left[\frac{1}{a} \ln \frac{\left(a-b \varepsilon_{(4)}^{(1)}\right) \varepsilon}{(a-b \varepsilon) \varepsilon_{(4)}^{(1)}}+\sigma(4)\right]
$$

where the initial condition was optimized and set as the fourth measured data point.

Both the predicted strain-stress curve and the stressstrain curve are shown in Figure 6, together with the 
experimental data. It is clear that excellent agreements were achieved by both the predicted curves, thus validating the proposed constitutive law based on the grey Verhulst model. Moreover, the carrying capacity of strain derived from Equation 16, is estimated as $a / b=0.68$, corresponding the infinite stress by Equation 17. This is also consistent with the fact that the minicomposites studied has the largest ultimate tensile strain of about $0.7 \%$ at the final failure.

\section{CONCLUSIONS}

A $\mathrm{C} / \mathrm{SiC}$ minicomposite was fabricated by chemical vapor infiltration and its microstructure and tensile behavior were investigated, as well as the associated damage evolution with the aid of acoustic emission technique. The largely nonlinear tensile behavior and the associated acoustic emission evolution of the minicomposites can be generally divided into three regimes including matrix cracking emergence, multiplication followed by matrix cracking saturation. Both the tensile behavior and the cumulative AE energy curve can be well predicted by the grey Verhulst models, indicating the nature of intraspecific competition for these matrix cracks in the minicomposites studied. Such relationships can be further applied to other ceramic composite systems to establish the constitutive law and optimize material parameters by the corresponding damage evolution.

\section{Acknowledgements}

The authors would like to acknowledge the financial support of the Scientific Research Foundation of Civil Aviation University of China under Grant No. 3122016B003, and Civil Aviation Administration of China under Grant No. MHRD20160105.

\section{REFERENCES}

1. Naslain R. (2004): Design, preparation and properties of non-oxide CMCs for application in engines and nuclear reactors: an overview. Composites Science Technology, 64, 155-170. doi:10.1016/S0266-3538(03)00230-6.
2. Yu H., Zhou X., Zhang W. (2013): Mechanical behavior of $\mathrm{SiCf} / \mathrm{SiC}$ composites with alternating $\mathrm{PyC} / \mathrm{SiC}$ multilayer interphases, Materials \& Design, 44, 320-324. doi:10.1016/ j.matdes.2012.07.073.

3. Wang Y., Zhang L., Cheng L. (2010): Comparison of inplane shear behaviors of 2-D plain woven $\mathrm{C} / \mathrm{SiC}$ composites. Ceramics-Silikaty, 54, 26-30.

4. Almansour A., Maillet E., Ramasamy S., Morscher G. N. (2015): Effect of fiber content on single tow SiC minicomposite mechanical and damage properties using acoustic emission. Journal of the European Ceramic Society, 35, 3389-3399. doi: 10.1016/j.jeurceramsoc.2015.06.001

5. Sauder C., Brusson A., Lamon J. (2010): Influence of interface characteristics on the mechanical properties of Hi-Nicalon type-S or Tyranno-SA3 fiber-reinforced $\mathrm{SiC} / \mathrm{SiC}$ minicomposites. International Journal of Applied Ceramic Technology, 7, 291-303. doi: 10.1111/j.17447402.2010.02485.x

6. Zhang S., Gao X., Dong H. (2017): Effects of gradual matrix crack closure on the constitutive behavior of $\mathrm{SiC} /$ $\mathrm{SiC}$ composites upon unloading. Ceramics International, 43, 1839-1842. doi:10.1016/j.ceramint.2016.10.142

7. Chateau C., Gelebart L., Bornert M. (2010): Experimental characterisation of damage in $\mathrm{SiC} / \mathrm{SiC}$ minicomposites. EPJ Web of Conferences, 6, 20002. doi:10.1051/epjconf/ 20100620002

8. Bueta E., Saudera C., Sornina D., Poissonnet S., Rouzaud J.-N., Vix-Guterl C. (2014): Influence of surface fibre properties and textural organization of apyrocarbon interphase on the interfacial shear stress of $\mathrm{SiC} / \mathrm{SiCminicomposites}$ reinforced with Hi-Nicalon S and Tyranno SA3 fibres. Journal of the European Ceramic Society, 34, 179-188. doi:10.1016/j.jeurceramsoc.2013.08.027

9. Zhang S., Gao X., Chen J., Dong H., Song Y. (2016): Strength model of the matrix element in $\mathrm{SiC} / \mathrm{SiC}$ composites. Materials \& Design, 101, 66-71. doi:10.1016/j. matdes.2016.03.166

10. Xu S. (2015): Model for evaluating the commercial banks financial risk with interval grey uncertain linguistic variables. Journal of Intelligent and Fuzzy Systems, 28, 767-773. doi:10.3233/IFS-141358

11. Liu S., Yang Y., Xie N., Forrest J. (2016): New progress of Grey System Theory in the new millennium. Grey Systems: Theory and Application, 6, 2-31. doi: 10.1108/GS-092015-0054

12. Liu S., Tao L., Xie N., Yang Y. (2016): On the New Model System and Framework of Grey System Theory. Journal of Grey System, 28, 1-15. 